\title{
VARIAÇÃO ESPACIAL E FORMAS GEOMÉTRICAS DAS REGENERAÇÕES NATURAIS DE ESPÉCIES DA FLORESTA ESTACIONAL SEMIDECIDUAL - PARQUE DO JEQUITIBÁ, CÁSSIA, MINAS GERAIS
}

\author{
Spatial Variation and Geometric Forms of Natural \\ Regenerations of Species of "Floresta Estacional Semidecidual" - \\ Jequitibá Park, Cassia, Minas Gerais
}

\author{
Sylvio Péllico Netto ${ }^{1}$ \\ Ulisses Silva da Cunha ${ }^{2}$ \\ Dennis Bernardi ${ }^{2}$ \\ Regina do Rocio de Andrade ${ }^{2}$ \\ Valmir Aparecido da Cunha ${ }^{2}$ \\ Eduardo Quadros da Silva ${ }^{2}$ \\ Norma Elizabeth Orue ${ }^{2}$ \\ Nilton Barth Filho ${ }^{2}$ \\ Saulo Henrique Weber ${ }^{2}$
}

\section{Resumo}

A presente pesquisa teve como principal objetivo estabelecer, para as espécies Bálsamo ou Cabriúva (Myrocarpus fron dosus Fr. Allem.), Peroba Rosa (Aspidosperma polyn euron Muell. Arg.), Sassafrás (Ocotea odorifera (Vell.) Rohwer), Guaritá (Astronium graveolensJacq.) e Pereira (Pratyciamusregnelli Benth.), cujos dados de regeneração natural foram coletados em um fragmento íntegro da Floresta Estacional Semidecidual, localizado na Fazenda Reata, município de Cássia, no Estado de Minas Gerais, denominado Parque do Jequitibá, com cerca de 90 hectares, uma comparação da variação espacial das plantas por meio de agregação dessas em formas triangulares, quadrangulares, pentagonais e hexagonais e compará-las com suas respectivas formas regulares equivalentes. A hipótese delineada neste trabalho partiu do pressuposto de que a distribuição espacial das árvores na floresta não segue regra geo métrica estrita, mas, como em todas as demais concepções biométricas, configura-se como aproximação às formas geométricas regulares. A proposta parte da formatação de uma rede espacial inicialmente com triângulos, depois com quadrados, com pentágonos e, finalmente, com hexágonos, utilizando-se sempre as mais próximas árvores posicionadas nos vértices dessas figuras geométricas. Os resultados mostraram que as formas triangulares e quadrangulares foram as que mais se aproximaram das formas geométricas naturais, porém o quadrado é a mais favorável a ser usado como modelo matemático, por facilitar as tratativas experimentais nas coletas de dados em campo e por apresentar melhor regularidade e proximidade entre a área média dos polígonos amostrais e a área média de equivalente polígonos regulares.

Palavras-chave: Distribuição espacial; Regeneração natural; Modelagem Matemática.

1 Professor Titular do Centro de Ciências Agrárias e Ambientais, da Pontifícia Universidade Católica do Paraná e professor sênior do Curso de Pós-Graduação em Engenharia da UFPR. E-mail: pellico.sylvio@pucpr.br. Rua Rocha Pombo, 791 CEP: 80.530-290, Curitiba, PR.

2 Doutorandos e Mestrandos do Curso de Pós-Graduação em Engenharia Florestal da UFPR. 


\section{Abstract}

The present research had as main objective to establish, for the species Bálsamo or Cabriúva (Myrocarpus frondosus Fr. Allem.), Rose Peroba (Aspidosperma polyneuron Muell. Arg.), Sassafrás (Ocotea odorifera (Vell.) Rohwer), Guaritá (Astronium graveolens Jacq.) e Pereira (Pratyciamus regnelli Benth.), whose data of natural regeneration had been collected in one natural stand of the "Floresta Estacional Semidecidual", located in the Reata Farm, county of Cássia, in the State of Minas Gerais, named Jequitibá Park, with about 90 hectares, a comparison of the plants spatial variation by means their aggregation in triangular, quadrangular, pentagonal and hexagonal forms and to compare them with their respective equivalent regular forms. The hypothesis delineated in this work had as main assumption that the spatial distribution of the trees in the forest does not follow strict geometric rule, but, as in all other biometrics conceptions, it is configured as approach to the regular geometric forms. The proposal started with the organization of a net initially made with triangles, after with squares, pentagons and, finally, with hexagons, using always the nearest trees located in the vertices to these geometric figures. The results had shown that the triangular and quadrangular forms had been the ones that came closer to the natural geometric forms, however the square is the most favorable form to be used as mathematical model, because of the facilities for collecting data in experimental field work and because it presents better regularity and proximity between the polygons average experimental area and their equivalent regular polygons area.

Keywords: Spatial distribution; Natural regeneration; Modeling.

\section{Introdução}

A pesquisa sobre variação espacial de árvores em uma floresta teve seu início com os estudos de König na Alemanha em 1835 (PRODAN, 1965). Destacam-se ainda outros autores como Pressler, 1865; Bauersachs, 1942; Merkle, 1948; Köhler, 1951/52; Stoffels, 1955; Weck, 1955; Essed, 1957; Hausburg, 1962 e Prodan, 1965, que estabeleceram relações matemáticas entre uma área média ocupada por uma árvore e o número de árvores por hectare existente na floresta. Para se chegar às áreas médias de ocupação das árvores na floresta, todos eles consideraram uma árvore como ponto amostra e as demais, vizinhas mais próximas dessa, como referência para a geração de uma superfície de ocupação espacial.

Tal conceito pode ser matematicamente expresso a partir da medida da distância euclidiana entre os centros de cada duas árvores mais próximas e, a partir daí, por simulação amostral, gerar áreas médias de ocupação das árvores. Genericamente, se for tomada a área de 1 hectare $(10.000$ $\mathrm{m}^{2}$ ), que pode ser denominada de A, a área de ocupação média de uma árvore como $\mathrm{a}_{\mathrm{i}} \mathrm{e}$, ainda, dependendo da densidade e da idade da floresta que $\mathrm{a}_{\mathrm{i}}$ se modifica, os autores acima referidos propuseram um fator de correção $\mathrm{k}_{\mathrm{i}}$, com valores alternativos para cada população amostrada, proveniente da variação de freqüência de ocorrência das espécies, resultando na relação genérica (01), para se obter o número médio de árvores $\mathrm{N}$ por hectare:

$$
N=k_{i} \frac{A}{a_{i}^{2}}
$$

Prodan (1965), usando essa relação e avaliando-a a partir de círculos concêntricos em sucessivas árvores mais próximas de um ponto amostral, considerou que, para o primeiro raio $\mathrm{a}_{0}$ tem-se $1 / 2$ árvore, para um segundo raio $\mathrm{a}_{1}$ tem-se $1+1 / 2$ árvore, para $\mathrm{a}_{2}$ tem-se $2+1 / 2$, e assim sucessivamente para as demais adicionais árvores, e propôs o seu método de amostragem conhecido como Amostragem de Seis Árvores, muito conhecido e aplicado na Alemanha, pela grande redução de tempo nos levantamentos florestais de povoamentos.

Conceitualmente, as relações acima concebidas pelos mencionados autores pressupuseram obter uma avaliação consistente para $a_{i}$, que pode ser conseguida de várias maneiras. A proposta delineada neste trabalho parte do pressuposto de que a distribuição espacial das árvores na floresta não segue regra geométrica estrita, mas, como em todas as demais concepções biométricas, configura-se como aproximações das formas geométricas regulares. A proposta parte da formatação de uma rede espacial inicialmente com triângulos, depois com quadrados, com pentágonos e, finalmente, com hexágonos, utilizando-se sempre as mais próximas árvores posicionadas nos vértices dessas figuras geométricas.

A hipótese estabelecida para tal amostragem espacial é de que as formas geométricas regulares mais simples, como o triangulo, tendem a ser 
mais próximas das formas espaciais naturais, do que as formas geométricas com maior número de lados.

\section{Metodologia}

Esta pesquisa foi efetuada no Parque do Jequitibá, localizado na Fazenda Reata, município de Cássia, MG. A área do Parque constitui-se de um fragmento de floresta natural de 90 hectares, remanescente da Floresta Estacional Semidecidual.

A comprovação da hipótese formulada foi efetuada a partir de dados experimentais de regenerações naturais das espécies Bálsamo ou Cabriúva (Myrocarpus frondosus Fr. Allem.), Peroba Rosa (Aspidosperma polyneuron Muell. Arg.), Sassafrás (Ocotea odorifera (Vell.) Rohwer), Guaritá (Astronium graveolens Jacq.) e Pereira (Pratyciamus regnelli Benth.) coletados em um fragmento íntegro da Floresta Estacional Semidecidual, localizado na Fazenda Reata, município de Cássia, no Estado de Minas Gerais, denominado Parque do Jequitibá, com cerca de 90 hectares.

Para a obtenção das formas geométricas, distâncias euclidianas foram tomadas entre duas árvores mais próximas e, a partir daí, sempre as interligando com a terceira mais próxima para a formação de triângulos, depois com a quarta mais próxima para a formação de quadriláteros, e assim sucessivamente para a formação dos pentágonos e, finalmente, dos hexágonos, sempre resultando em figuras adjacentes, sem árvores-ponto nos seus interiores e sem interseção de quaisquer lados.

Os dados foram gerados a partir de coordenadas absolutas $\left(\mathrm{x}_{\mathrm{i},} \mathrm{y}_{\mathrm{i}}\right)$ obtidas do levantamento de 100\% da regeneração natural, preliminarmente organizados em níveis correspondentes às diferentes espécies dentro de cada parcela e, posteriormente, em arquivos que permitiram a construção de gráficos no formato *. DXF para cada espécie e cada parcela, resultando em 12 arquivos utilizados em qualquer ambiente CAD (Computer Design Aided), o que permitiu georeferenciá-los nas quatro parcelas amostradas, cada uma de 1 hectare.

O processo construtivo das figuras geométricas, para cada uma das espécies, foi desenvolvido em ambiente CAD, usando-se o Software Microstation, começando pela formação dos triângulos, porque a partir desses se tornou mais fácil a geração das demais formas geométricas, valendo-se de um arquivo específico para cada uma delas, no âmbito de cada uma das parcelas.

A partir da simulação concluída, procedeu-se seqüencialmente ao cálculo de áreas e perímetros das figuras geométricas geradas, usando-se o Software Arcview. Do total das " $n$ " repetições por espécie, por parcela e para as figuras geométricas geradas, foram também estimados a média, a variância, o desvio padrão e o coeficiente de variação, para se conhecer o comportamento espacial das áreas ocupadas pelas regenerações naturais nos espaços amostrais georeferenciados.

As áreas médias resultantes de cada figura geométrica, para cada espécie, a partir da simulação, foram comparadas com as figuras geométricas regulares, obtidas usando-se os perímetros médios resultantes aplicados na formulação proposta por Selby (1974), conforme apresentado na Figura 1. 


\section{FIGURA 1 - Figura geométrica referência usada para a obtenção das áreas regulares em função dos perímetros médios.}

Figure 1 - Geometric figure used as reference for the attainment of the regular areas as function of the average perimeters.

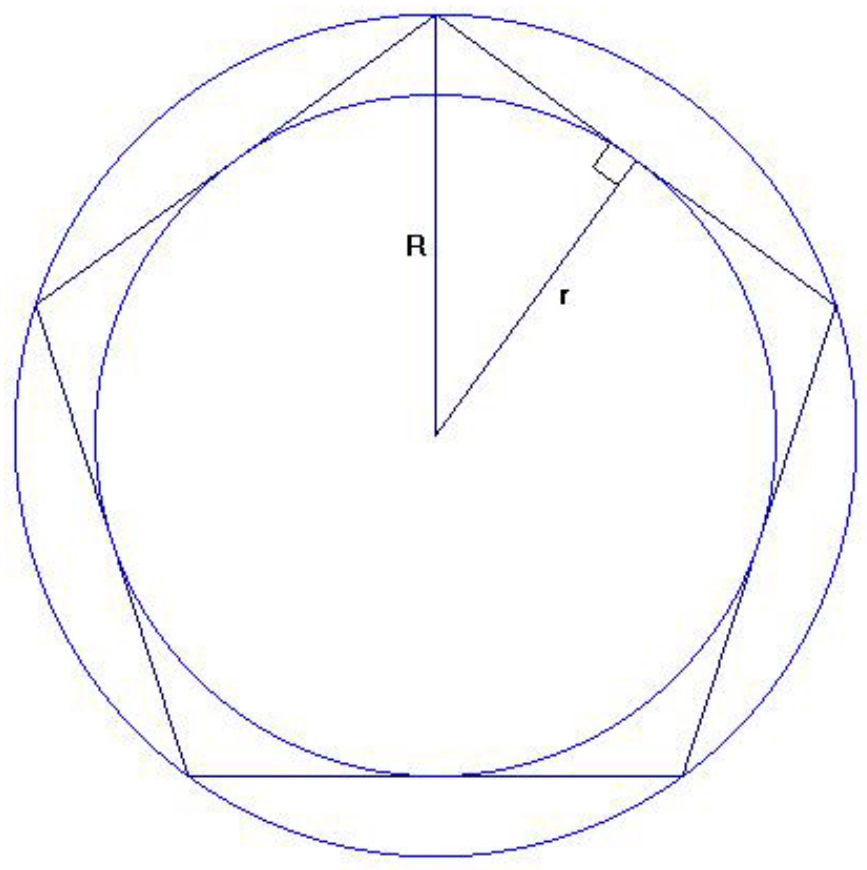

Por meio da Equação 2 obtém-se o ângulo $\theta$ interno da figura geométrica inscrita na circunferência $\mathrm{C} 1$, em função do seu número de lados $\mathrm{n}$.

$$
\theta=\left(\frac{n-2}{n}\right) 180^{\circ}
$$

A Equação 3 representa a relação entre 0 comprimento do lado do polígono $\mathrm{s}$, o raio $\mathrm{r}$ do círculo inscrito $\mathrm{C} 2$ à figura e seu número de lados n.

$$
s=2 r \cdot \operatorname{tg} \frac{180^{\circ}}{n}=2 R \cdot \operatorname{sen} \frac{180^{\circ}}{n}
$$

O perímetro p é calculado pelo produto entre o número de lados da figura n e seu comprimento s (Equação 4).

$$
p=n \cdot s
$$

A Equação 5 fornece a constante $\mathrm{k}$ a partir do número de lados $\mathrm{n}$ da figura, seus comprimentos s e seu número de lados.

$$
\begin{aligned}
& k=\frac{1}{4} n S^{2} \cot g \frac{180^{\circ}}{n}=n r^{2} \operatorname{tg} \frac{180^{\circ}}{n}= \\
& =\frac{1}{2} n R^{2} \operatorname{sen} \frac{180^{\circ}}{n}
\end{aligned}
$$

As Equações 6 e 7 são obtidas por meio de transformações da Equação 3.

$$
r=\frac{1}{2} s \cot g \frac{180^{0}}{n}
$$

$$
R=\frac{1}{2} s \cos \sec \frac{180^{\circ}}{n}
$$


onde

$\mathrm{k}=$ constante

$\mathrm{r}=$ apótema do pentágono ou raio do círculo inscrito ao pentágono

$\mathrm{R}=$ raio do círculo circunscrito ao pentágono

$\mathrm{s}=$ lado do polígono

$\theta=$ ângulo interno dos vértices

$\mathrm{p}=$ perímetro

$\mathrm{n}=$ número de lados

O cálculo de áreas foi obtido a partir de

$$
\mathrm{A}=\mathrm{k} \mathrm{s}^{2}
$$

te fórmula:

O valor de $\mathrm{k}$ foi obtido a partir da segun-

$$
k=\frac{1}{4} n \cdot s^{2} \operatorname{cotg} \frac{\pi}{\mathrm{n}}
$$

$$
\begin{array}{lll}
A=0,43301 & s^{2} & \text { Triângulo } \\
A=1,0000 & s^{2} & \text { Quadrado } \\
A=1,72048 & s^{2} & \text { Pentágono } \\
A=2,59808 & s^{2} & \text { Hexágono }
\end{array}
$$

\section{Resultados e discussão}

A partir da geração das coordenadas das regenerações naturais de cada espécie estudada, foram obtidas suas diferentes figuras geométricas. Ilustrações destes resultados para a espécie Peroba Rosa (Aspidosperma polyneuron Muell. Arg.) Apocynaceae, tomadas como destaque, estão apresentadas nas Figuras 2; 3; 4 e 5. 
Figura 2 - Formas geométricas triangulares relativas à distribuição espacial da regeneração natural da Peroba Rosa (Aspidosperma polyneuron Muell. Arg.) - Apocynaceae em 4 parcelas de 1 ha $(100 \mathrm{~m} \times 100 \mathrm{~m})$

Figure 2 - Spatial distribution of triangle geometric poligons of Rose Wood (Aspidosperma polyneuron Muell. Arg.) - Apocynaceae, in four plots of 1 hectare (100 m x 100 m)
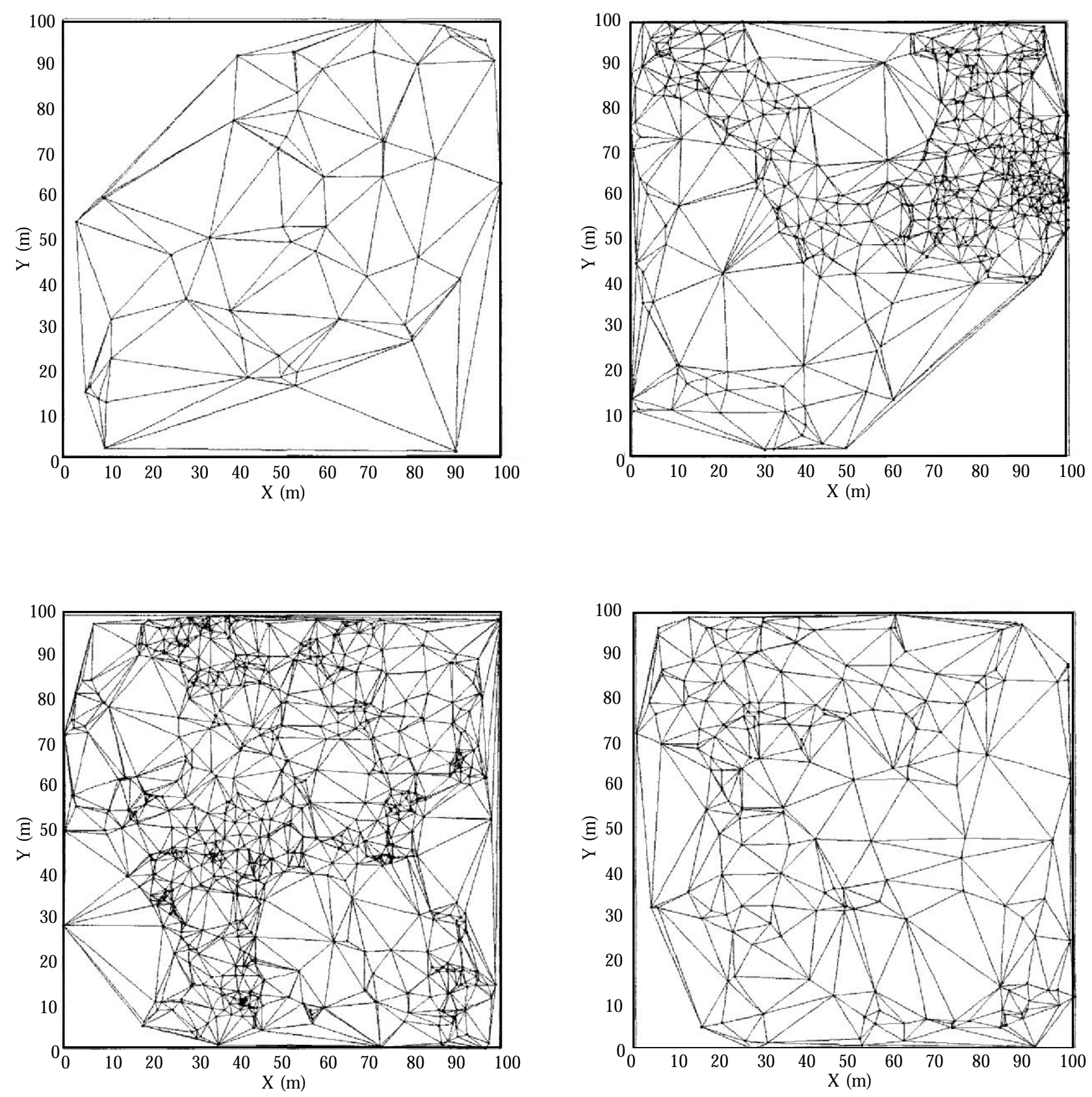
Figura 3 - Formas geométricas quadrangulares relativas à distribuição espacial da regeneração natural da peroba rosa (Aspidosperma polyneuron Muell. Arg.) - Apocynaceae em 4 parcelas de 1 ha $(100 \mathrm{~m} \times 100 \mathrm{~m})$

Figure 3 - Spatial distribution of quadrangular geometric poligons of rose wood (Aspidosperma polyneuron Muell. Arg.) - Apocynaceae, in four plots of 1 hectare (100 m x $100 \mathrm{~m})$
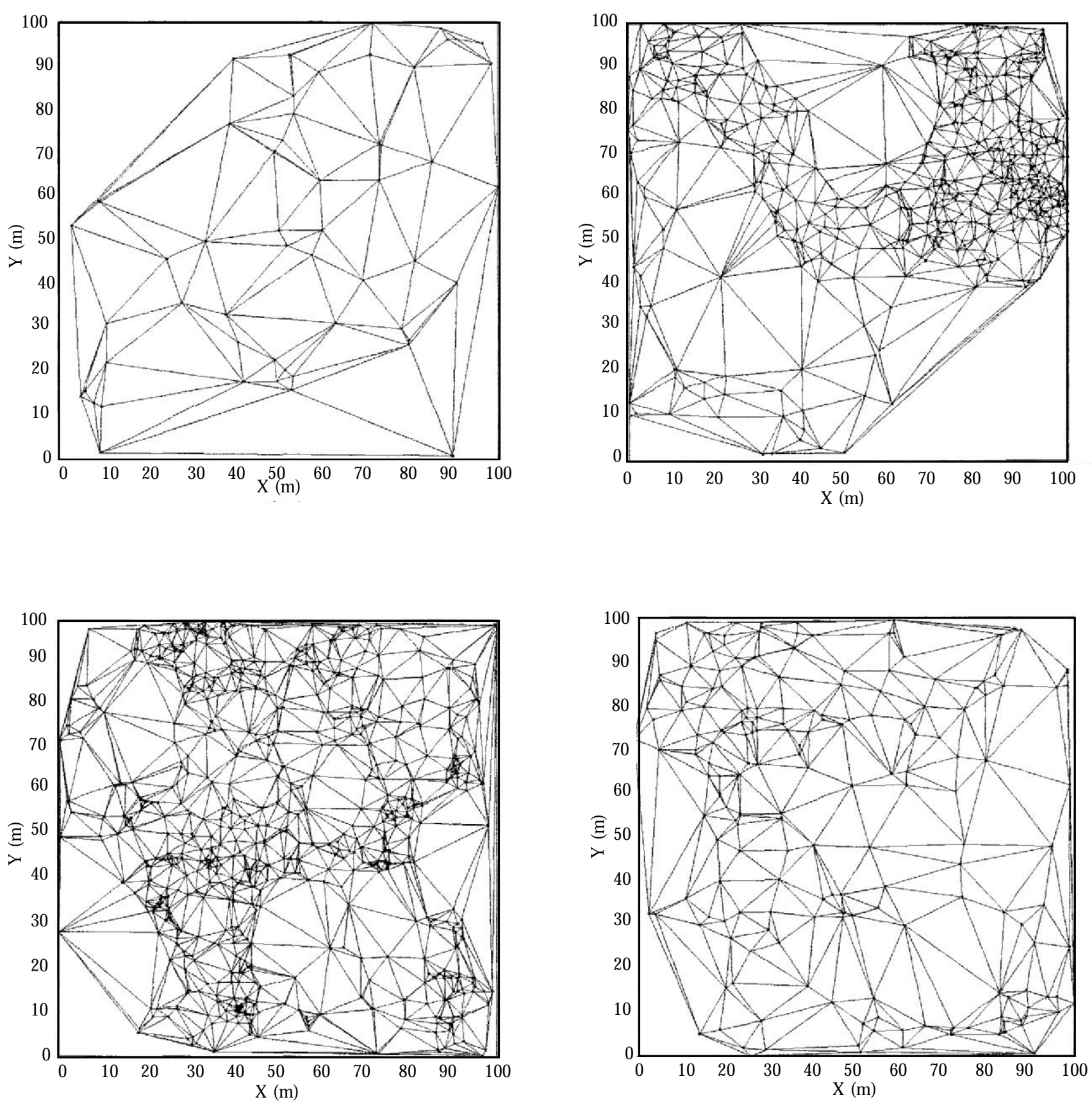
Figura 4 - Formas geométricas pentagonais relativas à distribuição espacial da regeneração natural da peroba rosa (Aspidosperma polyneuron Muell. Arg.) - Apocynaceae em 4 parcelas de 1 ha $(100 \mathrm{~m} \times 100 \mathrm{~m})$

Figure 4 - Spatial distribution of pentagonal geometric poligons of rose wood (Aspidosperma polyneuron Muell. Arg.) - Apocynaceae, in four plots of 1 hectare (100 m x 100 m).
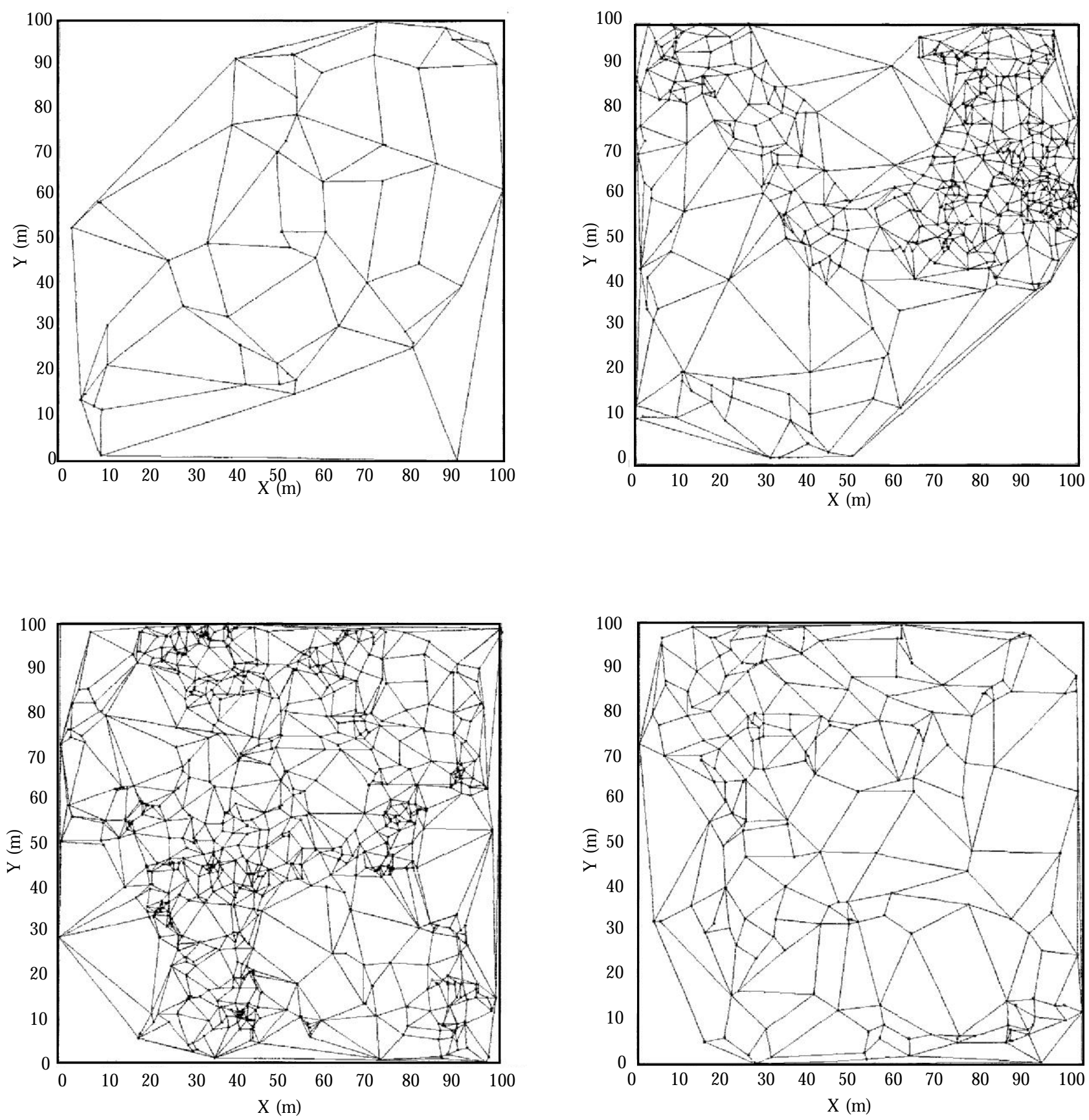


\section{Figura 5 - Formas geométricas hexagonais relativas à distribuição espacial da regeneração} natural da peroba rosa (Aspidosperma polyneuron Muell. Arg.) - Apocynaceae em 4 parcelas de 1 ha $(100 \mathrm{~m} \times 100 \mathrm{~m})$

Figure 5 - Spatial distribution of hexagonal geometric poligons of rose wood (Aspidosperma polyneuron Muell. Arg.) - Apocynaceae, in four plots of 1 hectare (100 m x $100 \mathrm{~m})$
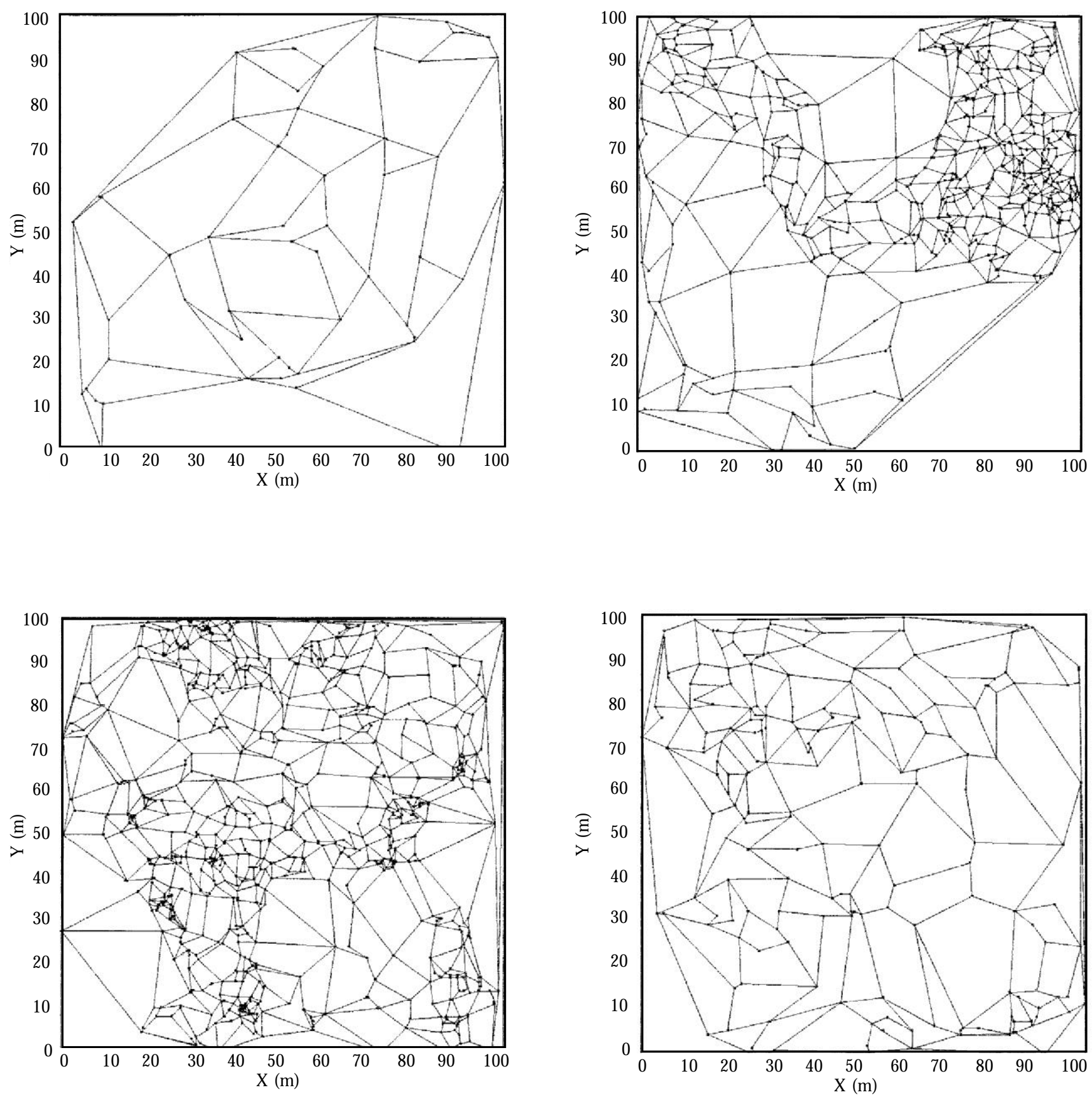
Os resultados processados para as diferentes espécies e diferentes formas geométricas estão apresentados nas Tabelas $1 ; 2 ; 3 ; 4 ; 5$ e 6.

Tabela 1 - Resultados dos estimadores estatísticos para a Cabriúva (Myrocarpus frondosus Fr. Allem.)

Table 1. Results of the statistical estimates for the specie Cabriuva (Myrocarpus frondosus Fr. Allem.)

\begin{tabular}{l|l|l|l|l}
\hline $\begin{array}{l}\text { FORMAS } \\
\text { GEOMÉTRICAS }\end{array}$ & $\begin{array}{l}\text { AREA AMOSTRAL } \\
\text { MÉDIA m }^{\mathbf{2}}\end{array}$ & $\begin{array}{l}\text { AREA REGULAR } \\
\text { MÉDIA m }^{\mathbf{2}}\end{array}$ & $\begin{array}{l}\text { DIFERENÇA } \\
\text { m }^{\mathbf{2}}\end{array}$ & $\begin{array}{l}\text { COEFICIENTE DE VARIAÇÃO } \\
\text { PARA ÁREAS AMOSTRAIS }\end{array}$ \\
\hline TRIÂNGULOS & 101,91 & 111,22 & 9,31 & $109 \%$ \\
QUADRILÁTEROS & 210,75 & 218,74 & 7,99 & $94 \%$ \\
PENTÁGONOS & 285,71 & 391,77 & 106,06 & $75 \%$ \\
HEXÁGONOS & 398,45 & 513,96 & 115,51 & $68 \%$ \\
\hline
\end{tabular}

Tabela 2 - Resultados dos estimadores estatísticos para a Peroba Rosa (Aspidosperma polyneuron Muell. Arg.)

Table 2. Results of the statistical estimates for the specie Rose Wood (Aspidosperma polyneuron Muell. Arg.)

\begin{tabular}{l|l|l|l|l}
\hline $\begin{array}{l}\text { FORMAS } \\
\text { GEOMÉTRICAS }\end{array}$ & $\begin{array}{l}\text { AREA AMOSTRAL } \\
\text { MÉDIA m }^{2}\end{array}$ & $\begin{array}{l}\text { AREA REGULAR } \\
\text { MÉDIA m }^{\mathbf{2}}\end{array}$ & $\begin{array}{l}\text { DIFERENÇA } \\
\text { m }^{\mathbf{2}}\end{array}$ & $\begin{array}{l}\text { COEFICIENTE DE VARIAÇAO } \\
\text { PARA ÁREAS AMOSTRAIS }\end{array}$ \\
\hline TRIÂNGULOS & 28,50 & 29,02 & 0,52 & $141 \%$ \\
QUADRILÁTEROS & 58,61 & 62,73 & 4,12 & $110 \%$ \\
PENTÁGONOS & 88,56 & 101,53 & 12,97 & $88 \%$ \\
HEXÁGONOS & 116,95 & 149,74 & 32,79 & $92 \%$ \\
\hline
\end{tabular}

Tabela 3 - Resultados dos estimadores estatísticos para a Sassafrás (Ocotea odorifera (Vell.) Rohwer)

Table 3. Results of the statistical estimates for the specie Sassafras (Ocotea odorifera (Vell.) Rohwer)

\begin{tabular}{l|l|l|l|l}
\hline $\begin{array}{l}\text { FORMAS } \\
\text { GEOMÉTRICAS }\end{array}$ & $\begin{array}{l}\text { AREA AMOSTRAL } \\
\text { MÉDIA m }^{2}\end{array}$ & $\begin{array}{l}\text { AREA REGULAR } \\
\text { MÉDIA m }^{2}\end{array}$ & $\begin{array}{l}\text { DIFERENÇA } \\
\text { m }^{\mathbf{2}}\end{array}$ & $\begin{array}{l}\text { COEFICIENTE DE VARIAÇÃO } \\
\text { PARA ÁREAS AMOSTRAIS }\end{array}$ \\
\hline TRIÂNGULOS & 173,70 & 252,23 & 78,53 & $87 \%$ \\
QUADRILÁTEROS & 363,54 & 693,93 & 330,39 & $57 \%$ \\
PENTÁGONOS & 576,90 & 948,68 & 371,78 & $43 \%$ \\
HEXÁGONOS & 741,97 & $1.485,08$ & 743,11 & $37 \%$ \\
\hline
\end{tabular}

Tabela 4 - Resultados dos estimadores estatísticos para o Guaritá (Astronium graveolens Jacq.)

Table 4. Results of the statistical estimates for the specie Guarita (Astronium graveolens Jaoq.)

\begin{tabular}{l|l|l|c|l}
\hline $\begin{array}{l}\text { FORMAS } \\
\text { GEOMÉTRICAS }\end{array}$ & $\begin{array}{l}\text { AREA AMOSTRAL } \\
\text { MÉDIA m }^{2}\end{array}$ & $\begin{array}{l}\text { AREA REGULAR } \\
\text { MÉDIA m }^{2}\end{array}$ & $\begin{array}{l}\text { DIFERENÇA } \\
\text { m }^{\mathbf{2}}\end{array}$ & $\begin{array}{l}\text { COEFICIENTE DE VARIACCÁO } \\
\text { PARA ÁREAS AMOSTRAIS }\end{array}$ \\
\hline TRIÂNGULOS & 36,46 & 40,43 & 3,97 & $121 \%$ \\
QUADRILÁTEROS & 73,20 & 81,68 & 8,48 & $108 \%$ \\
PENTÁGONOS & 109,43 & 136,02 & 26,59 & $96 \%$ \\
HEXÁGONOS & 147,15 & 195,93 & 48,78 & $89 \%$ \\
\hline
\end{tabular}


Tabela 5 - Resultados dos estimadores estatísticos para a Pereira (Pratyciamus regnelli Benth.)

Table 5. Results of the statistical estimates for the specie Pereira (Pratyciamus regnelli Benth.)

\begin{tabular}{|c|c|c|c|c|}
\hline $\begin{array}{l}\text { FORMAS } \\
\text { GEOMÉTRICAS }\end{array}$ & $\begin{array}{l}\text { ÁREA AMOSTRAL } \\
\text { MÉDIA m }{ }^{2}\end{array}$ & $\begin{array}{l}\text { ÁREA REGULAR } \\
\text { MÉDIA m² }\end{array}$ & $\begin{array}{l}\text { DIFERENÇA } \\
\mathbf{m}^{2}\end{array}$ & $\begin{array}{l}\text { COEFICIENTE DE VARIAÇÁO } \\
\text { PARA ÁREAS AMOSTRAIS }\end{array}$ \\
\hline TRIÂNGULOS & 351,35 & 491,72 & 140,37 & $70 \%$ \\
\hline QUADRILÁTEROS & 535,14 & 511,33 & 23,81 & $74 \%$ \\
\hline PENTÁGONOS & 856,42 & 812,55 & 43,87 & $54 \%$ \\
\hline HEXÁGONOS & $1.129,10$ & $1.015,38$ & 113,72 & $47 \%$ \\
\hline
\end{tabular}

Tabela 6 - Resultados dos estimadores estatísticos para a Jequitibá Rosa (Cariniana legalis (Raddi) Kuntze)

Table 6. Results of the statistical estimates for the specie Jequitiba Rosa (Cariniana legalis (Raddi) Kuntze)

\begin{tabular}{l|l|l|l|l}
\hline $\begin{array}{l}\text { FORMAS } \\
\text { GEOMÉTRICAS }\end{array}$ & $\begin{array}{l}\text { AREA AMOSTRAL } \\
\text { MÉDIA m }^{\mathbf{2}}\end{array}$ & $\begin{array}{l}\text { AREA REGULAR } \\
\text { MÉDIA m }^{\mathbf{2}}\end{array}$ & $\begin{array}{l}\text { DIFERENÇA } \\
\mathbf{m}^{\mathbf{2}}\end{array}$ & $\begin{array}{l}\text { COEFICIENTE DE VARIACCAO } \\
\text { PARA ÁREAS AMOSTRAS }\end{array}$ \\
\hline TRIÂNGULOS & 110,52 & 368,49 & 257,97 & $132,21 \%$ \\
QUADRILÁTEROS & 219,50 & 308,53 & 89,03 & $112,40 \%$ \\
PENTÁGONOS & 331,04 & 522,00 & 190,96 & $95,25 \%$ \\
HEXÁGONOS & 468,57 & 677,29 & 208,72 & $81,24 \%$ \\
\hline
\end{tabular}

Como se pode observar, em todas as espécies amostradas observa-se uma tendência para uma maior proximidade entre as áreas amostrais médias com as áreas regulares médias, avaliadas por suas respectivas diferenças.

Observa-se ainda que os coeficientes de variação, calculados para os tamanhos das áreas amostrais, tendem a decrescer à medida que aumenta o número de lado dos polígonos.
Uma síntese das diferenças obtidas para as diversas espécies e formas poligonais estudadas encontra-se na Tabela 7. Valores negativos significam que as médias obtidas para as áreas amostrais resultaram em valores menores que as médias de suas equivalentes formas regulares.

Tabela 7 - Diferença entre a área média dos polígonos amostrais e a área média de equivalente polígonos regulares.

Table 7. Difference between the average area of the sampled polygons and the average area of equivalent regular polygons.

\begin{tabular}{l|l|l|l|l|l|l|l|c}
\hline & Sassafrás & $\begin{array}{l}\text { Peroba } \\
\text { rosa }\end{array}$ & Pereira & Jequitibá & $\begin{array}{l}\text { Jequitibá } \\
\text { rosa }\end{array}$ & $\begin{array}{l}\text { Guaritá } \\
\text { branco }\end{array}$ & Canjerana & Bálsamo \\
\hline TRIÂNGULO & 78,53 & 0,52 & 140,36 & 257,97 & 57,81 & 3,97 & $-144,30$ & 9,31 \\
QUADRILÁTERO & 330,39 & 4,09 & $-23,82$ & 89,03 & 108,80 & 8,48 & $-308,56$ & 8,00 \\
PENTÁGONO & 371,91 & 12,94 & $-43,87$ & 190,96 & 96,31 & 26,59 & $-55,95$ & 106,03 \\
HEXÁGONO & 743,01 & 32,67 & $-113,72$ & 208,72 & 503,33 & 48,79 & $-91,34$ & 115,48 \\
\hline
\end{tabular}


Os resultados apresentados na Tabela 7 estão representados graficamente na Figura 6, para cada uma das formas e espécies estudadas.

FIGURA 6 - Representação gráfica das diferenças entre as áreas médias de padrões naturais e geométricos regulares (triângulos, quadriláteros, pentágonos e hexágonos) para regeneração natural de diferentes espécies da Floresta Estacional Semidecidual - Cássia, MG Figure 6 - Graphical representation of the differences between the average areas of natural and regular geometric standards (triangles, quadrilaterals, pentagons and hexagons) for natural regeneration of different species of the Floresta Estacional Semidecidual - Cassia, MG
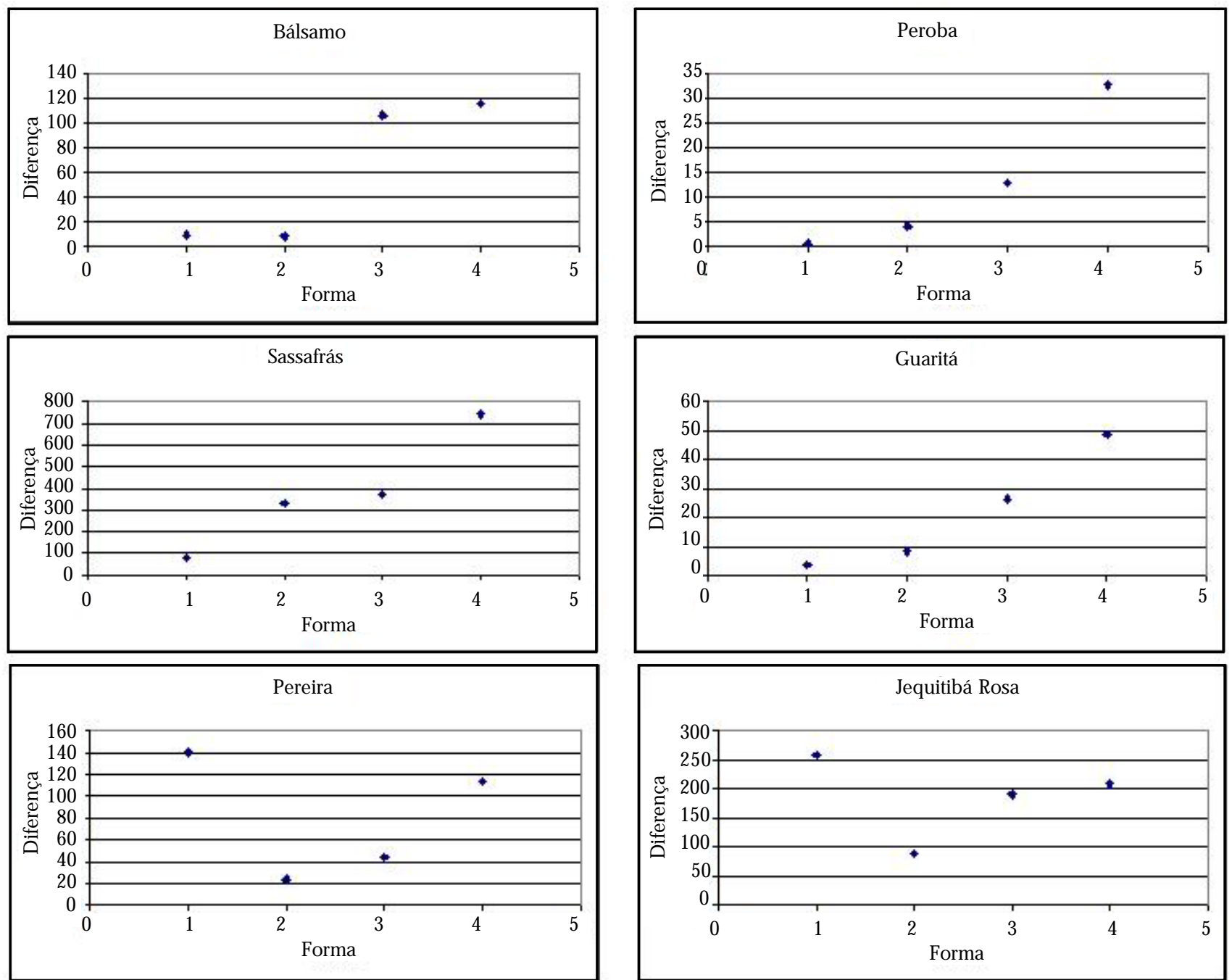

1 - Triângulo, 2 - Quadrilátero, 3 - Pentágono e 4 - Hexágono 


\section{Conclusões}

- O uso de figuras geométricas para conectar os mais próximos indivíduos das regenerações naturais de uma determinada espécie, tomando inicialmente o triângulo e ampliando-o até ao hexágono, demonstrou ser muito apropriado para compreender mais objetivamente o que ocorre no padrão da distribuição espacial das diferentes espécies;

- Dado o padrão espacial da maioria das espécies ser o agregado, a variabilidade das áreas das figuras geométricas com menor número de lados tende ser maior, decrescendo naquelas com maior número de lados, o que pode ser verificado nos resultados dos coeficientes de variação para cada uma das espécies estudadas;

- Dos resultados comparativos, usandose a tendência das respectivas diferenças entre 0 padrão natural e o padrão geométrico regular para cada figura geométrica estudada, pode-se concluir que duas formas geométricas regulares, o triângulo e o quadrado, são as que se mais aproximam do padrão geométrico natural, razão pela qual podem ser usadas na formulação teórica de métodos de amostragem. À medida que aumenta o número de lados nos polígonos (pentágonos e hexágonos) as formas geométricas regulares se distanciam do padrão geométrico natural, o que as tornam menos apropriadas para uso em modelagem matemática nos métodos amostrais;

- Entre as duas formas geométricas naturais que mais se aproximam das formas regulares, 0 quadrado é a mais favorável a ser usada como modelo matemático, por facilitar as tratativas expenmentais nas coletas de dados em campo e por apresentar melhor regularidade e proximidade entre a área média dos polígonos amostrais e a área média de equivalente polígonos regulares.

\section{Referências}

BAUERSACHS, E. Bestandsmassenaufnahme nach dem Mittelstammferfahren des zweitkleinsten Stammabstandes. ForstwissenschaftCentralblat, 1942. p. 182-186.

ESSED, F. E. Estimation of standing timber. Wageningen: Veenman, 1957.

HAUSBURG, H. Das Stammabstandsverfahren. Allgemeine Forst-und Jäggerzeitung. 1962.

KÖHLER, A. Vorratsermittlung in Buchenbeständen nach Stammdurchmesser und Stamm abstand. Allgemeine Forst-und Jäggerzeitung. 1951/1952

MERKLE, R. Die Holzvorratsaufnahme mit Hilfe der Stammabstände. Freiburger Diplomarbeit. 1948

PRESSLER, M. Das Gesetz der Stammbildung. Leipzig: [s. n.], P. 153. 1865.

PRODAN, M. Holzmesslehre. Frankfurt am Main: J. D. Sauerländer's Verlag.. 1965. p. 44.

SELBY, S. M. Standard mathematical tables. Cleveland, Ohio: Cranwood parkway, 1974. p. 706.

STOFFELS, A. Die Genauikeit der Bestimmung der Stammzahl pro Hektar durch Messung von Stammabständen. ForstwissenschaftCentralblat, n. 74. p. 211-219, 1955.

WECK, J. Vorratsermittlung durch Abstandsmessung. Zeitung für Weltforstwirtschaft. 1955. p. 24.

Recebido: 27/08/2004 Aprovado: 31/01/2005 